\title{
CONTINUITÉ/DISCONTINUITÉ DES CARRIÈRES DES JOURNALISTES FRANÇAIS ENCARTÉS. ÉTUDE DE DEUX COHORTES DE NOUVEAUX TITULAIRES DE LA CARTE DE PRESSE
}

\author{
Christine Leteinturier ${ }^{1}$
}

Cette étude propose d'étudier la question de la continuité des carrières des journalistes français à partir de la population constituée par les journalistes titulaires de la carte de presse. Les fichiers de la Commission de la carte d'identité des journalistes professionnels (CCIJP), étudiés sur 20 ans, mettent en lumière différents phénomènes propres au groupe professionnel journalistique, comme l'augmentation du nombre de sorties, particulièrement chez les plus jeunes actifs, la précarisation de l'emploi féminin, ainsi que la généralisation de carrières discontinues du point de vue des statuts et des employeurs. Ces constats mènent à considérer que la faible durée du passage par le journalisme (une moyenne de 15 ans sur l'ensemble des échantillons) situe le journalisme comme une séquence d'activité dans un espace professionnel élargi qu'il demeure difficile de cerner.

Avant d'aborder le phénomène des discontinuités dans les carrières des journalistes français, il importe de préciser notre objet d'étude et de rappeler quelques éléments concernant les sources disponibles et les méthodes utilisées.

1 Maître de conférences. Centre d'Analyse et de Recherche Interdisciplinaires sur les Médias (CARISM), Université Panthéon-Assas

Recherches en communication, $n^{\circ} 43$ - Article publié le 24/10/2016 
Les journalistes français « encartés » sont titulaires de la carte de presse délivrée par une commission paritaire la Commission de la Carte d'Identité des Journalistes Professionnels (CCIJP), élément constitutif du statut professionnel de mars 1935 créant la qualité de « journaliste professionnel »1. Son obtention et/ ou son renouvellement sont liés à la situation professionnelle de l'intéressé en lien avec la définition juridique du statut de journaliste professionnel telle que donnée par l'article L.7611-3 du Code du travail : «Le journaliste professionnel est celui qui a pour occupation principale, régulière et rétribuée, l'exercice de sa profession dans une ou plusieurs publications quotidiennes ou périodiques ou dans une ou plusieurs agences de presse et qui en tire le principal de ses ressources $»$.

Cette carte est annuelle, renouvelée chaque année. Si elle reste un « sésame » pour accéder à des espaces spécifiques nécessaires à la collecte de l'information, la carte n'est pas obligatoire pour l'exercice du métier de journaliste ni pour l'accès à certains avantages statutaires consentis à cette profession ${ }^{2}$. Toutefois, elle joue partiellement un rôle « social » en cas de chômage. En effet, la carte de presse peut être attribuée, pour deux années consécutives maximum, à des journalistes sans emploi à deux conditions. La première est qu'ils aient eu la carte de presse antérieurement au moins pendant 24 mois consécutifs et la seconde qu'ils soient déclarés à Pôle emploi pour entrer dans le dispositif du chômage indemnisé. Nous reviendrons plus particulièrement sur le chômage comme composante des carrières des journalistes français.

Les quelques 36000 journalistes titulaires de la carte de presse, reconnus journalistes professionnels au sens de la loi, ne représentent donc qu'une fraction de l'ensemble des professionnels qui se disent journalistes et dont l'INSEE estime le nombre à quelques 50000 personnes. La carte de presse peut être perçue comme un dispositif de régulation du groupe des

1 Voir le site de la CCIJP pour toutes les informations concernant l'origine de la carte et les conditions de son attribution et de son renouvellement : http://www.ccijp.net

2 Par exemple l'abattement fiscal supplémentaire consenti aux journalistes d'un montant annuel de $7650 €$ n'est pas lié à la détention de la carte de presse (Bastin, 2014). 
journalistes, mais elle est surtout un dispositif de légitimation (Leteinturier, 2012) qui contribue alors à diviser le groupe entre les encartés et les non encartés. Les titulaires de la carte de presse représentent les plus insérés dans le cadre du statut professionnel de 1935, ceux pour lesquels la carte est précisément un symbole d'appartenance à un groupe distinct et distinctif.

Du fait de son activité, la CCIJP constitue depuis sa création en 1936 des archives très précieuses pour la connaissance des journalistes français. L'Institut Français de Presse dispose depuis 25 ans d'une expertise de travail sur ces archives, en particulier à travers trois grandes enquêtes successives qui ont permis de construire progressivement une méthodologie (Leteinturier, 2015) d'analyse des carrières des journalistes titulaires de la carte de presse. En effet, la CCIJP nous permet d'accéder aux dossiers individuels des journalistes, sur une base échantillonnée par cohortes de nouveaux entrants dits NTCP-Nouveaux titulaires de la carte de presse. C'est ainsi que nous avons constitué déjà trois cohortes de NTCP pour les années 1990, 1998 et 2008 :

Tableau 1 : échantillon analysé : effectifs des NTCP 1990, 1998 et 2008 (CCIJP/ ANR-AMMEJ)

\begin{tabular}{|c|c|c|c|}
\hline & 1990 & 1998 & 2008 \\
\hline $\begin{array}{l}\text { Ensemble des premières } \\
\text { demandes de carte }\end{array}$ & 2200 & 1880 & 2004 \\
\hline Échantillons NTCP & $\begin{array}{c}498 \\
(21 \%)\end{array}$ & $\begin{array}{c}430 \\
(23 \%)\end{array}$ & $\begin{array}{c}537 \\
(26,7 \%) \\
\end{array}$ \\
\hline
\end{tabular}

Ces travaux sont donc largement empiriques, fournissant des données inédites permettant de mieux comprendre certains mécanismes à l'œuvre dans la construction de leurs carrières par les journalistes. Il faut préciser qu'il ne s'agit pas d'une analyse précise du marché de l'emploi journalistique dans l'espace médiatique français global. Pour cela, il faudrait accéder à d'autres sources, en particulier les effectifs globaux des entreprises médiatiques et leur répartition par catégories d'emploi (Renoux, 2011, 2014, 2015). 
Cette source, si elle est une " aubaine » pour la sociologie du journalisme, présente des limites qu'il faut rappeler. Elle ne couvre qu'une partie de la population journalistique française, celle qui demande et obtient la carte. Les données sont fournies via un dispositif déclaratif, les documents sont remplis par le demandeur. La Commission peut effectuer des contrôles sur les éléments déclarés quand elle a un doute soit sur l'emploi lui-même (qualification, revenu) soit sur l'entreprise (s'agit-il bien d'un média), car elle collecte également les déclarations collectives des employeurs eux-mêmes. Il y a donc un préjugé de véracité des principaux éléments fournis. De fait, c'est donc moins la fiabilité des données qui pose question que les lacunes que génère le dispositif administratif de la Commission, en particulier dans le cas qui nous occupe, à savoir les discontinuités dans les carrières des journalistes encartés. En effet, quand un journaliste ne renouvelle pas sa carte, on ignore pourquoi. Quand l'interruption est temporaire, il peut à son retour fournir des précisions, mais c'est rare. Il est donc impossible de saisir, à travers les dossiers de la CCIJP, ce que fait le journaliste en dehors de l'activité qu'il déclare sur la période de détention de la carte.

Les discontinuités saisies à partir des dossiers de la CCIJP vont alors être partielles, et n'être, pour certaines, que partiellement documentées. Ces discontinuités sont le fruit de différents aléas qui peuvent survenir au long de la carrière que l'on envisage alors comme un continuum. Dans l'idéal et dans une perspective plutôt interactionniste, il faut, comme le rappelle Michel Lallement (2007), pouvoir saisir « les articulations entre organisation du travail, gestion des mobilités et trajectoires biographiques ». Le journaliste joue individuellement un rôle important, mais aussi l'ensemble des formes de socialisation qu'il traverse et qui peuvent influer sur sa mobilité interne dans l'organisation ou sur sa mobilité externe, d'organisations en organisations, du secteur des médias vers d'autres secteurs. Dans un contexte de crise économique durable, comme celle que traversent les médias français depuis plus de trente ans, les stratégies des entreprises vont également être à l'origine de modifications des trajectoires professionnelles 
et pas seulement parce que les réorganisations internes peuvent aboutir à des licenciements et donc à du chômage. Elles peuvent aussi être saisies comme des opportunités d'évolution pour aller vers d'autres secteurs ou pour investir autrement le marché du travail, par exemple en devenant soi-même entrepreneur. Celles que l'on va pouvoir cerner à travers les informations collectées auprès de la CCIJP vont essentiellement concerner la mobilité, interne ou externe, le passage par le chômage et la sortie du groupe, sans que l'on puisse en donner une cause précise, en dehors de celles qui vont être liées au refus de renouvellement de la carte de presse. De même, pour ceux qui sortent du fichier de la CCIJP, il est très difficile de savoir ce qu'ils deviennent.

\section{Les carrières des NTCP de 1990 et 1998 : caractéristiques générales}

Insistons sur le fait que les éléments d'analyse des carrières des journalistes disent des choses sur les marchés d'emploi, mais dans une perspective " en creux » : seules des enquêtes auprès des entreprises et plus particulièrement des recruteurs (rédacteurs en chef et services $\mathrm{RH}$ ) permettent de comprendre la réalité des emplois existants dans les entreprises (Marchetti \& Ruellan, 2001).

La construction de la carrière des journalistes encartés à partir de leurs dossiers individuels de la CCIJP s'organise autour de trois séquences professionnelles. La première correspond à l'ensemble des activités exercées, sous quelque forme que ce soit y compris les stages, avant la première demande de carte. La deuxième est la séquence d'activité qui va permettre l'obtention de la carte, dont la durée est variable ; la CCIJP elle-même demande la justification de trois mois continus d'activité, mais ce sont souvent des séquences plus longues qui sont déclarées. La troisième correspond à la carrière elle-même, c'est-à-dire la séquence qui suit l'obtention de la première carte et qui est validée par la succession des renouvellements annuels. Cette carrière est spécifiée par les caractéristiques sociodémographiques classiques des titulaires : sexe, âge, domicile, formation générale et 
professionnelle, média d'exercice, qualification, statut, revenu et la mention éventuelle d'autres activités parallèles. Cette méthode d'analyse a été mise en œuvre à partir de la deuxième enquête IFP par Valérie Devillard (2001) qui a réalisé la première analyse des carrières des journalistes entrés en 1990 sur 8 ans. L'obtention d'un contrat avec l'ANR en 2008, nous a conduit à relancer cette analyse en prolongeant l'étude des deux cohortes de NTCP 1990 et 1998 jusqu'à 2008, en y ajoutant une nouvelle cohorte pour 2008 (Leteinturier, 2014). Une nouvelle étude' ${ }^{1}$ a débuté en 2015 qui reprend les trois cohortes précédentes jusqu'à 2015, en en ajoutant une nouvelle, les nouveaux titulaires de la carte de presse 2013.

\subsection{Dans quels médias ont-ils fait carrière?}

Tableau 2 : Répartition de l'ensemble des carrières pour chaque cohorte par types de médias (CCIJP / ANR - AMMEJ)

\begin{tabular}{|c|c|c|c|c|}
\hline & \multicolumn{2}{|c|}{1990} & \multicolumn{2}{|c|}{1998} \\
\hline & Effectifs & $\%$ & Effectifs & $\%$ \\
\hline Carrières presse & 359 & 72,2 & 284 & 65,9 \\
\hline $\begin{array}{l}\text { Carrières presse + } \\
\text { autres médias }\end{array}$ & 49 & 9,9 & 45 & 10,4 \\
\hline $\begin{array}{l}\text { Carrières ADV (radio } \\
\text { et/ou TV) }\end{array}$ & 66 & 13,3 & 71 & 16,5 \\
\hline $\begin{array}{l}\text { Carrières ADV + } \\
\text { autres médias }\end{array}$ & 2 & 0,4 & 11 & 2,6 \\
\hline Carrières Agences & 20 & 4,0 & 19 & 4,4 \\
\hline Carrières web & 1 & 0,2 & 1 & 0,2 \\
\hline Total effectifs NTCP & 498 & 100 & 428 & 100 \\
\hline
\end{tabular}

Par ailleurs, la mobilité entre médias est très faible puisque les parcours plurimédias sont très peu nombreux : seulement 61 (12, $2 \%)$ NTCP 1990 et $56(13 \%)$ NTCP 1998 ont travaillé dans plusieurs médias. Encore peut-on penser qu'il s'agit, pour une grande part, de pigistes exerçant dans plusieurs médias.

1 Voir sur le site de la CPNEF Audiovisuel : http://www.cpnef-av.fr/les-etudes/ insertion-des-jeunes-journaliste 


\subsection{Sous quel statut?}

Le statut d'exercice joue son rôle dans la construction des parcours.

Tableau 3 : Répartition part statuts des NTCP 1990 et 1999 (CCIJP / ANR-AMMEJ)

\begin{tabular}{|l|c|c|c|}
\hline & $\begin{array}{c}\mathbf{1 9 9 0} \\
\text { (à 11 ans) }\end{array}$ & $\begin{array}{c}\mathbf{1 9 9 8} \\
\text { (à 11 ans) }\end{array}$ & $\begin{array}{c}\mathbf{1 9 9 0} \\
\text { (à 18 ans) }\end{array}$ \\
\hline Exclusivement CDI & 21,1 & 15,4 & 17,5 \\
\hline Exclusivement pigistes & 1,0 & 2,3 & 0,4 \\
\hline $\begin{array}{l}\text { Exclusivement Pigiste/ } \\
\text { CDD }\end{array}$ & 1,0 & 2,3 & 0,4 \\
\hline Exclusivement CDD & 0,0 & 0,0 & 0,0 \\
\hline $\begin{array}{c}\text { Carrières sous statuts } \\
\text { hétérogènes sans } \\
\text { chômage }\end{array}$ & 13,1 & 11,0 & 11,6 \\
\hline $\begin{array}{r}\text { Total carrières } \\
\text { continues }\end{array}$ & 36,2 & 31,0 & 29,9 \\
\hline $\begin{array}{l}\text { Carrières avec } \\
\text { chômage et/ou } \\
\text { périodes inconnues }\end{array}$ & 27,9 & 14,0 & 15,3 \\
\hline Sortants sur la période & 35,9 & 55,0 & 54,8 \\
\hline $\begin{array}{r}\text { Total carrières } \\
\text { discontinues }\end{array}$ & 63,8 & 69,0 & 70,1 \\
\hline Rappel effectifs & 498 & 430 & 498 \\
\hline
\end{tabular}

Les positions statutaires stables tout au long du parcours, les CDI - contrats à durée indéterminée, sont globalement minoritaires puisqu'elles ne représentent que 21 à $17 \%$ des situations, selon les cohortes observées. Les carrières continues se font majoritairement en CDI ou via des parcours hétérogènes sans chômage, mais qui vont aboutir, à un moment ou à un autre, à une position en CDI, ces deux types de parcours concernant la moitié des effectifs pour chaque groupe. Les parcours continus de pigistes sont rares. Pour la cohorte 1990, ils représentent $1 \%$ de l'ensemble à 11 ans, mais 2,3\% pour la cohorte 1998. Le chômage 
est assez largement présent dans les carrières : il concerne $28 \%$ de la cohorte 1990 à 11 ans, plus que la cohorte 1998 à 11 ans qui n'est qu'à $14 \%$. Mais nous avons observé que cette cohorte 1998 avait connu plus de départs définitifs, certains rapidement après l'obtention de la première carte.

1.3. Des carrières continues minoritaires sur la période étudiée...

Les carrières continues complètes ne concernent véritablement qu'une minorité des titulaires de la carte de presse : $40 \%$ pour la cohorte 1990 et $30 \%$ pour la cohorte 1998 , observation à 11 ans. Sur 18 ans, les carrières continues des NTCP 1990 ont baissé de 10 points pour atteindre $30 \%$ de la cohorte initiale ! Il y a donc bien lieu de parler de discontinuités importantes dans les carrières de ces groupes de journalistes.

Tableau 4 : Types de carrières des NTCP 1990 et 1999 en \% de l'ensemble de chaque cohorte (CCIJP / ANR-AMMEJ)

\begin{tabular}{|r|r|r|r|}
\hline Types de Carrières & NTCP 1990 & NTCP 1998 & NTCP 1990 \\
\hline en \% & \multicolumn{1}{c|}{ à 11 ans } & \multicolumn{1}{c|}{ à 11 ans } & \multicolumn{1}{c|}{ à 18 ans } \\
\hline $\begin{array}{r}\text { Carrières continues } \\
\text { complètes }\end{array}$ & \multicolumn{1}{|c|}{38,9} & 31,1 & \multicolumn{1}{c|}{29,5} \\
\hline dans même média & 32,5 & 26,1 & 25,8 \\
\hline dans médias différents & 6,4 & 5,0 & 3,7 \\
\hline Carrières discontinues & 61,1 & 68,9 & \multicolumn{1}{|c|}{70,5} \\
\hline $\begin{array}{r}\text { Complètes avec } \\
\text { chômage ou sortie } \\
\text { temporaire }\end{array}$ & 25,0 & 14,0 & 15,3 \\
\hline avec sorties définitives & 36,1 & 54,9 & 55,2 \\
\hline Rappel effectifs & 498 & 430 & 498 \\
\hline
\end{tabular}

La faible mobilité intermédia est confirmée aussi pour les carrières continues conformément à la tendance générale déjà observée : $6,4 \%$ et $5 \%$ pour les cohortes 1990 et 1998 à 11 ans, et baisse à 3,7 \% pour les NTCP 1990 à 18 ans. La mobilité 
intermédia serait-elle un handicap pour faire une carrière continue et longue?

Les sorties temporaires ou le passage par le chômage, des discontinuités sans sorties définitives, ont concerné à 11 ans le quart des NTCP 1990 et $14 \%$ des NTCP 1998 : à 18 ans, $15 \%$ des NTCP 1990. Les carrières des NTCP 1998 sont donc différentes de celles de leurs aînés de 1990, globalement plus courtes. Il y a moins de discontinuité dans leurs carrières, mais en revanche une proportion nettement plus importante de sorties définitives (54, 9\% pour les NTCP 1998 à 11 ans et seulement $36 \%$ pour les NTCP 1990 à 11 ans). Peut-on faire l'hypothèse d'une moindre résistance, d'une moindre obstination face aux aléas du secteur? Ou s'agit-il d'une volonté plus affirmée de consolider sa situation professionnelle même en dehors du journalisme plutôt que de « ramer » dans un secteur devenu fragile ? Nous verrons que la confrontation au chômage pèse lourd sur la poursuite des carrières, et dans ce contexte, la CCIJP peut jouer un rôle régulateur du fait de sa capacité à maintenir dans le cadre de la carte de presse des journalistes temporairement sans emploi.

\subsection{Répartition par médias des carrières continues sans chômage ni discontinuités}

La part des carrières complètes continues est variable selon les médias, car elle est liée à la répartition des journalistes par types de médias. Elle correspond globalement à ce qui a été déjà été observé en termes de répartition 1990 et 1998 des nouveaux journalistes par médias.

Tableau 5 : les carrières complètes continues selon les cohortes : répartition par médias en $\%$ de l'ensemble des carrières complètes (CCIJP / ANR-AMMEJ)

\begin{tabular}{|l|c|c|c|}
\hline & $\begin{array}{c}\text { NTCP 1990 } \\
\text { à 11 ans }\end{array}$ & $\begin{array}{c}\text { NTCP 1998 } \\
\text { à 11 ans }\end{array}$ & $\begin{array}{c}\text { NTCP 1990 } \\
\text { à 18 ans }\end{array}$ \\
\hline $\begin{array}{l}\text { Presse \& presse } \\
\text { + autres médias }\end{array}$ & 83,4 & 71,3 & 82,4 \\
\hline
\end{tabular}




\begin{tabular}{|c|c|c|c|}
\hline $\begin{array}{l}\text { ADV \& ADV } \\
+ \text { autres médias }\end{array}$ & 14,0 & 23,9 & 14,9 \\
\hline Agences & 2,6 & 4,8 & 2,7 \\
\hline $\begin{array}{l}\text { Total effectif } \\
\text { carrières complètes }\end{array}$ & 319 & 193 & 225 \\
\hline $\begin{array}{l}\text { Part en \% des } \\
\text { carrières complètes }\end{array}$ & 64,0 & 45,0 & 45,2 \\
\hline Rappel effectifs NTCP & 498 & 428 & 498 \\
\hline
\end{tabular}

Le croisement statut / média des carrières exclusivement en CDI confirme que la presse écrite est le média qui offre les positions les plus stables. À l'intérieur de l'ensemble presse, ce sont la presse quotidienne régionale et la presse spécialisée qui accueillent le plus grand nombre de carrières continues. La presse nationale est très peu présente dans les carrières de ces deux cohortes. Les autres secteurs, en particulier l'audiovisuel, sont très peu fournisseurs de carrières complète en CDI, car les modalités d'accès à ces univers professionnels sont différentes : le passage par des sas d'attente (piges et CDD) est très largement répandu et l'accès au CDI peut être très tardif.

Tableau 6 : les médias d'exercice des NTCP ayant une carrière complète en CDI (CCIJP / ANR-AMMEJ)

\begin{tabular}{|l|c|c|c|}
\hline & $\begin{array}{c}\text { NTCP 1990 } \\
\text { à 11 ans }\end{array}$ & $\begin{array}{c}\text { NTCP 1998 } \\
\text { à 11 ans }\end{array}$ & $\begin{array}{c}\text { NTCP 1990 } \\
\text { à 18 ans }\end{array}$ \\
\hline Presse & 90 & 50 & 77 \\
\hline Audiovisuel & 6 & 9 & 3 \\
\hline Agences & 2 & 5 & 1 \\
\hline Web & 2 & 1 & 2 \\
\hline Rappel effectif CDI & $105(21 \%)$ & $66(15 \%)$ & $87(17,4 \%)$ \\
\hline Rappel effectif NTCP & 498 & 428 & 498 \\
\hline
\end{tabular}

1.5. Une forte proportion de « sortants » définitifs des fichiers de la CCIJP

Les « sorties » définitives des comptes de la CCIJP montrent une nette accélération entre 1990 et 2008 pour les cohortes 1990 
et 1998. Ces résultats corroborent les constats faits par Valérie Devillard dans le cadre de l'enquête IFP 1999.

Graphique 1 : Les rythmes de sortie des NTCP 1990 \& 1998 entre 1990 et 2008 (CCIJP / ANR-AMMEJ)

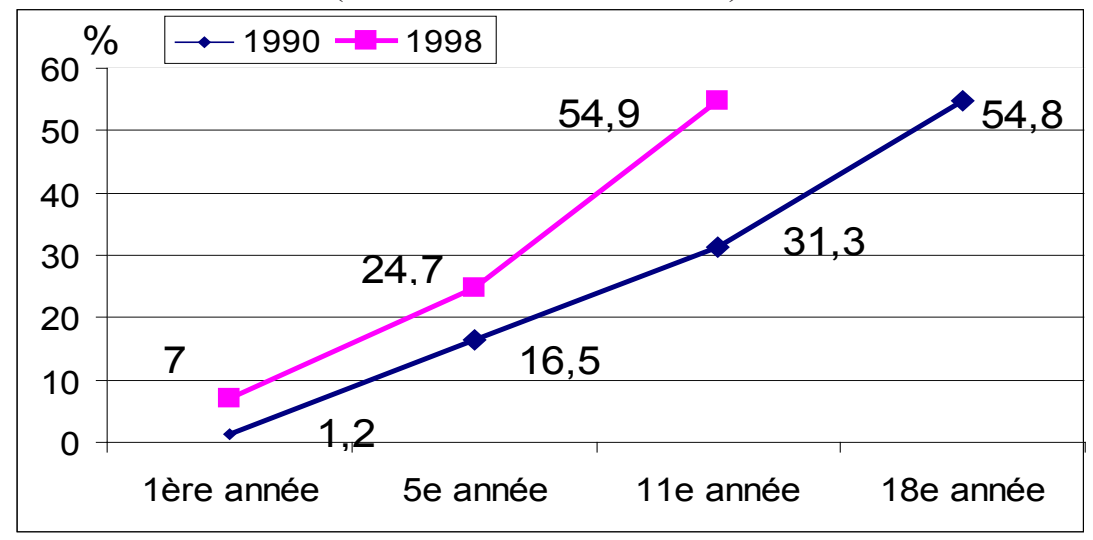

En 18 ans, la cohorte des NTCP 1990 a perdu plus de la moitié de ses effectifs. Sur 11 ans, la cohorte 1990 n'avait perdu que le tiers de ses effectifs, celle de 1998 en a perdu la moitié. Le rythme des sorties des fichiers de la CCIJP s'accélère donc entre 1990 et 1998, montrant ainsi une dégradation de l'emploi de journalistes dans les médias au cours de cette décennie.

\section{Influence des caractéristiques sociodémographiques sur les carrières}

\subsection{Des effets de génération}

Le phénomène des sorties précoces touche des journalistes entrés plus tardivement dans les fichiers de la CCIJP, et donc, pour la majorité d'entre eux cela signifie une entrée tardive en journalisme, après une autre activité. L'âge peut être un facteur explicatif quand un journaliste n'accède à la carte de presse qu'après 40 ou 45 ans. Mais pour ceux qui entrent entre 30 et 40 ans, il s'agit aussi d'une difficulté à s'adapter à ce nouveau secteur d'activité, à ses contraintes et à ses aléas. 
Tableau 7 : nouveaux titulaires de la carte de presse 1990 \& 1998 : profils des carrières en \% de l'effectif de chaque cohorte (CCIJP/ ANR-AMMEJ)

\begin{tabular}{|l|c|c|c|c|}
\hline En \% & \multicolumn{2}{|c|}{1990} & \multicolumn{2}{c|}{1998} \\
\hline $\begin{array}{c}\text { Types de } \\
\text { carrières }\end{array}$ & $\begin{array}{c}\text { Jeunes } \\
(<\mathbf{3 1} \text { ans })\end{array}$ & $\begin{array}{c}\text { Tardifs } \\
(=>\mathbf{3 1} \text { ans })\end{array}$ & $\begin{array}{c}\text { Jeunes } \\
(<\mathbf{3 1} \text { ans })\end{array}$ & $\begin{array}{c}\text { Tardifs } \\
(=>\mathbf{3 1} \text { ans })\end{array}$ \\
\hline $\begin{array}{l}\text { Carrières } \\
\text { Complètes }\end{array}$ & 68,4 & 52,7 & 52,4 & 38,6 \\
\hline $\begin{array}{l}\text { Sortants du } \\
\text { fichier CCIJP }\end{array}$ & 31,6 & 47,3 & 47,6 & 61,4 \\
\hline Total & 100,0 & 100,0 & 100,0 & 100,0 \\
\hline
\end{tabular}

2.2 Des effets de genre : une fragilité croissante de l'emploi féminin

Dans chacune des deux cohortes observées, les femmes sont moins nombreuses que les hommes, autour de $47 \%$ : le processus de féminisation semble marquer le pas. Elles sont aussi proportionnellement moins nombreuses à faire des carrières complètes, donc un peu plus à avoir des carrières interrompues. C'est ainsi, qu'à échéance de 11 ans de carrière, l'écart entre les hommes et les femmes, pour les carrières complètes est de 2 points pour 1990, mais de 8 points pour 1998. La fragilité de l'emploi féminin en journalisme augmenterait donc entre 1990 et 1998 alors même que les femmes sont globalement plus diplômées que les hommes.

Tableau 8 : Les carrières complètes de journalistes encartés selon les statuts d'exercice : comparaison femmes : hommes

\begin{tabular}{|l|c|c|c|c|c|c|}
\cline { 2 - 7 } \multicolumn{1}{c|}{} & \multicolumn{1}{c}{1990} & A & 11 ans & \multicolumn{1}{c|}{1998} & \multicolumn{1}{c|}{11 ans } \\
\cline { 2 - 7 } \multicolumn{1}{c|}{} & F & H & $\begin{array}{c}\text { Ensem- } \\
\text { ble }\end{array}$ & F & H & $\begin{array}{c}\text { Ensem- } \\
\text { ble }\end{array}$ \\
\hline $\begin{array}{l}\text { Exclusive-ment } \\
\text { CDI }\end{array}$ & 46 & 59 & 105 & 22 & 44 & 66 \\
\hline $\begin{array}{l}\text { Pigistes étendus } \\
\text { (Piges + CDD) }\end{array}$ & 2 & 3 & 5 & 2 & 8 & 10 \\
\hline
\end{tabular}




\begin{tabular}{|l|c|c|c|c|c|c|}
\hline $\begin{array}{l}\text { Parcours } \\
\text { hétérogènes }\end{array}$ & 19 & 49 & 70 & 28 & 30 & 57 \\
\hline Avec chômage & 47 & 32 & 79 & 18 & 15 & 33 \\
\hline $\begin{array}{l}\text { Avec période } \\
\text { inconnue }\end{array}$ & \multicolumn{1}{c|}{34} & 28 & 60 & 9 & 18 & 27 \\
\hline $\begin{array}{r}\text { Total carrières } \\
\text { complètes }\end{array}$ & $\begin{array}{r}148 \\
(46 \\
\%)\end{array}$ & $\begin{array}{r}171 \\
(54 \\
\%)\end{array}$ & 319 & $\begin{array}{r}79 \\
(40 \%)\end{array}$ & $\begin{array}{r}114 \\
(60 \%)\end{array}$ & 193 \\
\hline $\begin{array}{r}\text { Rappel effectifs } \\
\text { NTCP }\end{array}$ & 237 & 261 & 498 & 201 & 227 & 428 \\
\hline
\end{tabular}

En effet, entre 1990 et 1998, elles sont moins nombreuses à faire des carrières complètes : $46 \%$ pour la cohorte 1990 à 11 ans et seulement $40 \%$ pour la cohorte 1998 à 11 ans. Elles sont d'ailleurs globalement proportionnellement moins nombreuses à faire des carrières complètes en CDI : $43 \%$ pour la cohorte 1998 à 11 ans et $33 \%$ pour la cohorte 1998. Par ailleurs, elles sont constamment plus nombreuses que les hommes parmi les chômeurs et les périodes inconnues.

Tableau 9 : la répartition des carrières complètes par médias et par genres pour les NTCP 1990 et 1998 (CCIJP / ANR-AMMEJ)

\begin{tabular}{|l|c|c|c|c|c|c|}
\cline { 2 - 7 } \multicolumn{1}{c|}{} & \multicolumn{2}{c|}{ NTCP 1990 à 11 ans } & \multicolumn{2}{c|}{ NTCP 1998 à 11 ans } \\
\cline { 2 - 7 } \multicolumn{1}{c|}{} & F & H & $\begin{array}{c}\text { Ensem- } \\
\text { ble }\end{array}$ & F & H & $\begin{array}{c}\text { Ensem- } \\
\text { ble }\end{array}$ \\
\hline $\begin{array}{l}\text { Presse \& } \\
\text { presse et autres } \\
\text { médias }\end{array}$ & 130 & 133 & 263 & 68 & 80 & 148 \\
\hline $\begin{array}{l}\text { ADV \& ADV + } \\
\text { autres médias }\end{array}$ & 16 & 27 & 43 & 17 & 29 & 46 \\
\hline Agences & 1 & 6 & 7 & 2 & 8 & 10 \\
\hline $\begin{array}{l}\text { Total effectif } \\
\text { carrières } \\
\text { complètes }\end{array}$ & 147 & 166 & 313 & 87 & 117 & 204 \\
\hline $\begin{array}{l}\text { Part en \% des } \\
\text { carrières }\end{array}$ & 62,0 & 63,6 & 62,9 & 43,3 & 51,5 & 47,7 \\
\hline $\begin{array}{l}\text { Total effectif } \\
\text { carrières } \\
\text { interrompues }\end{array}$ & 90 & 95 & 185 & 114 & 110 & 224 \\
\hline
\end{tabular}




\begin{tabular}{|r|r|r|r|r|r|r|}
\hline $\begin{array}{r}\text { Part en \% } \\
\text { des carrières }\end{array}$ & 38,0 & 36,4 & 37,1 & 56,7 & 48,5 & 52,3 \\
\hline $\begin{array}{r}\text { Rappel } \\
\text { effectifs NTCP }\end{array}$ & 237 & 261 & 498 & 201 & 227 & 428 \\
\hline
\end{tabular}

Elles sont proportionnellement un peu plus nombreuses en presse pour la cohorte 1990 environ $50 \%$, mais moins nombreuses en 1998 (46\%).

Tableau 10 : Les positions des journalistes à l'obtention de la carte de presse : comparaison femmes / hommes

\begin{tabular}{|l|c|c|c|c|c|c|}
\hline En \% & \multicolumn{3}{|c|}{ Cohorte 1990 } & \multicolumn{3}{c|}{ Cohorte 1998 } \\
\hline & F & H & $\begin{array}{c}\text { Ensem- } \\
\text { ble }\end{array}$ & F & H & $\begin{array}{c}\text { Ensem- } \\
\text { ble }\end{array}$ \\
\hline CDI & 62,4 & 61 & 61,7 & 47,3 & 45,1 & 46,2 \\
\hline CDD & 9,3 & 10,4 & 9,9 & 24,6 & 15,3 & 20,2 \\
\hline CQ & 0,8 & 0,4 & 0,6 & 2 & 3,1 & 2,6 \\
\hline PIGISTE & 27,4 & 27,8 & 27,6 & 26,1 & 35,4 & 31 \\
\hline
\end{tabular}

À l'obtention de la carte de presse, la situation statutaire des NTCP a beaucoup changé entre les deux cohortes. Pour la cohorte 1990, le CDI prévalait pour la majorité, puisque plus de $60 \%$ en avaient un. EN 1998, le CDI à l'entrée est devenu minoritaire $(46,2 \%)$ et ce sont les contrats précaires (piges, CDD et CQ) qui dominent avec 53,8 \%. En revanche les positions respectives des femmes et des hommes restent stables entre les deux cohortes. Elles sont un peu plus nombreuses à être salariées en CDI. Leurs positions dans les contrats plus précaires ont évolué entre les deux cohortes; elles sont devenues plus nombreuses à entrer avec $\mathrm{CDD}$ alors que les hommes sont plus nombreux à entrer comme pigistes.

\section{Le diplôme en journalisme, un rendement à long terme.}

Les diplômés en journalisme connaissent des trajectoires relativement différentes selon les cohortes. Ceci s'explique en fait par la croissance régulière des effectifs de diplômés en journalisme dans chacune des cohortes : $30 \%$ pour la cohorte 1990 et $43 \%$ pour la cohorte 1998. L'augmentation du niveau 
de formation professionnelle, qui se poursuit de façon régulière jusqu'à aujourd'hui, conduit à s'interroger sur la « protection » que fournit le diplôme.

Nous avons observé, dans un précédent travail (Leteinturier, 2010) sur la formation des journalistes, que le diplôme en journalisme ne conduit pas nécessairement à une entrée facile dans le métier.

Tableau 11 : Position statutaire à l'obtention de la carte de presse : ensemble et diplômés en journalisme

\begin{tabular}{|l|c|c|c|c|}
\cline { 2 - 5 } \multicolumn{1}{c|}{} & $\mathbf{1 9 9 0}$ & & $\mathbf{1 9 9 8}$ & \\
\hline en \% & $\begin{array}{c}\text { Dipl. } \\
\text { Journ. }\end{array}$ & Ensemble & $\begin{array}{c}\text { Dipl. } \\
\text { Journ. }\end{array}$ & Ensemble \\
\hline CDI & 46,67 & 61,7 & 37,04 & 46,2 \\
\hline CDD & 19,33 & 9,9 & 27,51 & 20,2 \\
\hline CQ & 2,00 & 0,6 & 5,82 & 2,6 \\
\hline PIGE & 32,00 & 27,6 & 29,63 & 31 \\
\hline
\end{tabular}

Les diplômés en journalisme occupent curieusement des positions plus précaires que l'ensemble des nouveaux titulaires de la carte de presse. En 1990, ils sont plus de $53 \%$ à occuper des positions précaires et plus de $62 \%$ en 1998. Le diplôme, comme nous l'avions déjà observé dans notre travail sur les nouveaux titulaires de la carte de presse 2008, ne présente pas de garantie particulière pour obtenir une position stable à l'obtention de la carte de presse. Plusieurs raisons expliquent ce phénomène. Les diplômés en journalisme, mieux informés que les autres, demandent leur carte relativement vite après la sortie de la formation, sans attendre d'avoir une position stable : trois mois de pige ou de contrat salarié suffisent, et en général ils décrochent facilement un CDD à la sortie de l'école. Ils s'orientent également plus volontiers vers le secteur de l'audiovisuel qui embauche majoritairement en contrats à durée déterminée, et pendant longtemps avant de titulariser ses journalistes en CDI. Enfin, le passage par ces statuts précaires ne leur semble sans doute pas très risqué, du fait même qu'ils estiment que leur diplôme leur fournit un avantage compétitif. 
Tableau 12 : Taux de sorties des NTCP 1990 et 1998 : ensemble et diplômés en journalisme (CCIJP / ANR-AMMEJ)

\begin{tabular}{|l|c|c|c|c|}
\hline \multicolumn{1}{|c|}{ En \% } & \multicolumn{2}{|c|}{ NTCP 1990 } & \multicolumn{2}{c|}{ NTCP1998 } \\
\hline & Dipl. Jism & Ensemble & Dipl. Jism & Ensemble \\
\hline $\mathbf{1}^{\text {ère }}$ année & 6,6 & 1,6 & 3,7 & 8,4 \\
\hline $\mathbf{5}^{\mathrm{e}}$ année & 15,3 & 20,3 & 17,6 & 25,2 \\
\hline $\mathbf{1 0}^{\mathrm{e}}$ année & 24 & 34,3 & 45,4 & 43,5 \\
\hline $\mathbf{1 7}^{\mathrm{e}}$ année & 44 & 55 & & \\
\hline $\begin{array}{c}\text { Rappel des } \\
\text { effectifs }\end{array}$ & & & & \\
\hline
\end{tabular}

L'intérêt du diplôme se mesure au fil du temps, au fil de la carrière. Pour la cohorte 1990, le diplôme en journalisme représente une réelle protection puisqu'à partir de la $5^{\mathrm{e}}$ année d'exercice l'écart de la part des sortants entre les diplômés et les non-diplômés augmente de façon régulière : 5 points à la $5^{\mathrm{e}}$ année, 10 points à $10^{\mathrm{e}}$ et à $1 \mathrm{a} 17^{\mathrm{e}}$. Le diplôme en journalisme est donc bien un élément positif de résistance aux aléas des trajectoires pour la cohorte 1990. Pour la cohorte 1998 la situation est plus complexe. Il consolide la situation en début de carrière puisque les taux de sortie sont moindres pour la première et la cinquième année. Pour la $10^{\mathrm{e}}$ année, l'écart entre les deux groupes est faible, les diplômés sortent à peu près comme l'ensemble du groupe. L'un des facteurs explicatifs est la situation difficile des médias classiques face à l'arrivée du numérique et la forte attractivité des innovations numériques, même si toutes ne permettent pas d'obtenir la carte de presse.

En termes de trajectoire, on constate également une différence entre les deux cohortes et un positionnement plutôt atypique des diplômés en journalisme. Ils sont nettement moins nombreux proportionnellement dans les parcours stables et plus nombreux dans les parcours hétérogènes. Cela confirme le rôle de protection relative du diplôme qui permet de résister même dans des situations statutaires délicates, positions parfois choisies, car offrant une plus grande liberté d'action. Il faut également noter que les diplômés se dirigent aussi plus volontiers vers l'audiovisuel où 
les structures d'emploi reposent beaucoup sur le contrat à durée déterminée dit CCD d'usage, constamment renouvelé.

Tableau 13 : les parcours type des NTCP 1990 et 1998 : comparaison ensemble et diplômés en journalisme (CCIJP / ANR-AMMEJ)

\begin{tabular}{|c|c|c|c|c|}
\hline \multirow{2}{*}{$\begin{array}{l}\text { Parcours types } \\
\text { en } \%\end{array}$} & 1990 & 1990 & 1998 & 1998 \\
\hline & $\begin{array}{l}\text { Dipl. } \\
\text { Jism }\end{array}$ & Ensemble & $\begin{array}{l}\text { Dipl. } \\
\text { Jism }\end{array}$ & Ensemble \\
\hline $\begin{array}{l}\text { Exclusivement } \\
\text { salariés (CDI \& } \\
\text { CDD) }\end{array}$ & 33,2 & 42,3 & 36,1 & 39,5 \\
\hline donc exclusiv. $C D I$ & 27,8 & 38,7 & 24,6 & 32,3 \\
\hline $\begin{array}{l}\text { Pigistes étendus } \\
\text { (Piges \& CDD) }\end{array}$ & 6,6 & 10 & 4,7 & 11,5 \\
\hline $\begin{array}{l}\text { Parcours } \\
\text { hétérogènes (avec } \\
\text { chômage) }\end{array}$ & 60,2 & 47,7 & 59,2 & 49 \\
\hline Rappel des effectifs & & & & \\
\hline
\end{tabular}

Le diplôme présente également un avantage indéniable pour progresser dans la carrière et prendre des responsabilités d'encadrement d'équipes. C'est ainsi que les diplômés sont plus nombreux à devenir cadres pour la cohorte 1990. Le phénomène est moins visible pour la cohorte 1998 qui a été observée sur une durée plus courte. Toutefois, ceci confirme globalement la valeur de l'investissement que représente le passage par une formation au journalisme.

Tableau 14 : évolution de carrière des NTCP 1990 et 1998 : ensemble et diplômés en journalisme (CCIJP / ANR-AMMEJ)

\begin{tabular}{|l|r|r|r|r|}
\hline & \multicolumn{2}{|c|}{1990} & \multicolumn{2}{c|}{1998} \\
\hline $\begin{array}{l}\text { Évolution } \\
\text { base / cadre }\end{array}$ & Dipl. Jism & Ensemble & Dipl. Jism & Ensemble \\
en \% & 48,34 & 39,6 & 25,65 & 23,7 \\
\hline
\end{tabular}

Le marché français de la formation des journalistes est fortement concurrentiel, mais segmenté en deux pôles. Le premier, considéré comme le plus prestigieux, est constitué par 
les écoles dites « formations reconnues par la profession" ${ }^{1}$ » qui sont aujourd'hui quatorze. Elles répondent à des critères précis tant sur le plan pédagogique que sur les conditions d'accès et elles attirent beaucoup, car elles ont noué, au fil du temps, des partenariats de formation avec en particulier les grands médias nationaux et régionaux (télévision, radio et presse quotidienne). À l'exception de deux IUT, elles recrutent pratiquement toutes à bac +3 pour délivrer des diplômes de niveau Master 2. Le second groupe est constitué de formations diverses, une majorité d'écoles privées dont le niveau de recrutement se situe souvent au niveau du baccalauréat, un nombre croissant de formations universitaires de niveau Master 2 et quelques formations universitaires de niveau L3. Les différences de profil de formation des étudiants issus de ces deux types de cursus sont importantes, ce qui conduit également à des types de carrières différenciés.

\section{Causes des discontinuités temporaires ou de sorties définitives}

4.1. Le refus de carte de presse prononcé par la CCIJP et ses causes

Tableau 15 : La part des refus dans le travail de la CCIJP entre 2009 et 2011 (Source CCIJP. ANR-AMMEJ)

\begin{tabular}{|l|c|c|c|}
\hline & $\mathbf{2 0 0 9}$ & $\mathbf{2 0 1 0}$ & $\mathbf{2 0 1 1}$ \\
\hline $\begin{array}{l}\text { Total cartes } \\
\text { délivrées }\end{array}$ & 37390 & 37007 & 36815 \\
\hline Total refus & $507(1,2 \%)$ & $679(1,8 \%)$ & $560(1,5 \%)$ \\
\hline Premières cartes & 2024 & 1822 & 1854 \\
\hline Refus 1ères cartes & $142(28 \%)$ & $159(23,4 \%)$ & $137(24,5 \%)$ \\
\hline Renouvellements & 35366 & 35185 & 34961 \\
\hline $\begin{array}{l}\text { Refus } \\
\text { renouvellements }\end{array}$ & $365(72 \%)$ & $520(76,6 \%)$ & $423(75,5 \%)$ \\
\hline
\end{tabular}

La part des refus reste faible au regard du nombre de dossiers traités aboutissant à une réponse favorable, entre 1 et $2 \%$ selon les années (Leteinturier, 2014). De même la distribution des refus

1 Voir les sites suivants : http://www.cnmj.fr/basedocumentaire/cpnej/; http:// cpnej.free.fr/ 
entre refus de $1^{\text {ère }}$ carte et refus de renouvellement reste à peu près stable sur les trois années observées : autour d'un quart pour les refus de première carte et des trois quarts pour les refus de renouvellements.

Tableau 16 : les « refusés » 2010 : répartition, en \%, des causes de refus $\{37$ dossiers sur 679$\}$ (CCIJ/ ANR-AMMEJ)

\begin{tabular}{|c|c|c|c|c|c|c|}
\hline \multirow{2}{*}{$\begin{array}{l}\text { Causes des refus } \\
2010\end{array}$} & \multicolumn{3}{|c|}{ Ensemble des refus } & \multicolumn{3}{|c|}{$\begin{array}{c}\text { Primo-demandeurs } \\
\text { refusés }\end{array}$} \\
\hline & Total & H. & F. & Total & H. & $\mathbf{F}$ \\
\hline \multicolumn{7}{|c|}{ Liées à l'individu } \\
\hline $\begin{array}{l}\text { Activités non } \\
\text { journalistiques }\end{array}$ & 16,40 & 12,82 & 19,95 & 20,68 & 10,19 & 29,46 \\
\hline $\begin{array}{l}\text { Statut } \\
\text { professionnel } \\
\text { non conforme }\end{array}$ & 15,11 & 16,78 & 13,07 & 24,47 & 25,93 & 23,26 \\
\hline $\begin{array}{l}\text { Ressources } \\
\text { insuffisantes }\end{array}$ & 14,04 & 13,52 & 14,68 & 10,97 & 14,81 & 7,75 \\
\hline Chômage & 11,98 & 12,59 & 11,24 & 0 & 0 & 0 \\
\hline $\begin{array}{l}\text { Qualification } \\
\text { non reconnue }\end{array}$ & 3,81 & 3,73 & 3,90 & 8,86 & 8,33 & 9,30 \\
\hline Incompatibilité & 3,78 & 4,43 & 2,98 & 2,53 & 3,70 & 1,55 \\
\hline $\begin{array}{l}\text { Honoraire } \\
\text { (retraité) }\end{array}$ & 2,35 & 1,63 & 3,21 & 0 & 0 & 0 \\
\hline $\begin{array}{l}\text { N'exerce plus } \\
\text { l'activité }\end{array}$ & 1,67 & 2,10 & 1,15 & 0,42 & 0,93 & 0 \\
\hline $\begin{array}{l}\text { Emission } \\
\text { ADV non } \\
\text { journalistigue }\end{array}$ & 1,55 & 0,93 & 2,29 & 2,11 & 0,93 & 3,10 \\
\hline $\begin{array}{l}\text { Activité } \\
\text { et revenus } \\
\text { irréguliers }\end{array}$ & 1,16 & 1,17 & 1,15 & 1,69 & 2,78 & 0,78 \\
\hline Directeur & 0,74 & 1,17 & 0,23 & 2,11 & 3,70 & 0,78 \\
\hline $\begin{array}{l}\text { Absence de } \\
\text { justification de } \\
\text { règlement }\end{array}$ & 0,46 & 0,47 & 0,46 & 0 & 0 & 0 \\
\hline \multicolumn{7}{|c|}{ Liées à l'entreprise } \\
\hline $\begin{array}{l}\text { Entreprise non } \\
\text { reconnue }\end{array}$ & 14,89 & 14,69 & 15,14 & 19,83 & 20,37 & 19,38 \\
\hline
\end{tabular}




\begin{tabular}{|l|r|r|r|r|r|r|}
\hline $\begin{array}{l}\text { Hors presse } \\
\text { majoritaire }\end{array}$ & 10,84 & 12,59 & 8,72 & 5,06 & 7,41 & 3,10 \\
\hline $\begin{array}{l}\text { Ne relève pas } \\
\text { de la législation } \\
\text { française }\end{array}$ & 1,60 & 1,40 & 1,83 & 1,27 & 0,93 & 1,55 \\
\hline
\end{tabular}

Les refus touchent majoritairement les pigistes et toutes les formes de contrats précaires, soit environ $36 \%$ des refus prononcés en 2010, du fait en particulier de l'insuffisance des ressources tirées du journalisme. Le second groupe le plus touché par les refus est celui des chômeurs, aux alentours de $20 \%$ des refus. Les titulaires de CDI qui se voient refuser la carte occupent pour la plupart une activité journalistique non reconnue.

4.2. Le poids du chômage, des licenciements et des situations précaires

Un journaliste au chômage peut continuer de détenir la carte de presse à condition qu'il l'ait eue, de façon continue, pendant au moins deux ans (24 mois). Il doit aussi fournir des preuves d'indemnisation par le Pôle emploi ; souvent le journaliste au chômage explique sa situation soit par un licenciement aboutissant à une rupture du contrat de travail (CDI) soit par le renouvellement d'un contrat temporaire, de type piges ou contrat à durée déterminée (CDD).

L'évolution de l'effectif global des chômeurs titulaires de la carte de presse passe de 830 en 1990 à 1576 en 2012, soit un quasi-doublement en 22 ans.

Tableau 17 : Évolution du nombre des chômeurs encartés entre 1990 et 2012 (CCIJP / IFP-CARISM)

\begin{tabular}{|l|c|c|c|}
\hline & Effectif total & $\begin{array}{c}\text { Effectif } \\
\text { chômeur }\end{array}$ & $\begin{array}{c}\text { Part des } \\
\text { chômeurs (\%) }\end{array}$ \\
\hline $\mathbf{1 9 9 0}$ & 26614 & 830 & 0,5 \\
\hline $\mathbf{1 9 9 9}$ & 30510 & 1100 & 1,7 \\
\hline $\mathbf{2 0 0 8}$ & 37307 & 1342 & 3,6 \\
\hline $\mathbf{2 0 1 2}$ & 36815 & 1576 & 4,3 \\
\hline
\end{tabular}

Quel est l'impact réel de ce chômage sur le cours de la carrière ? 


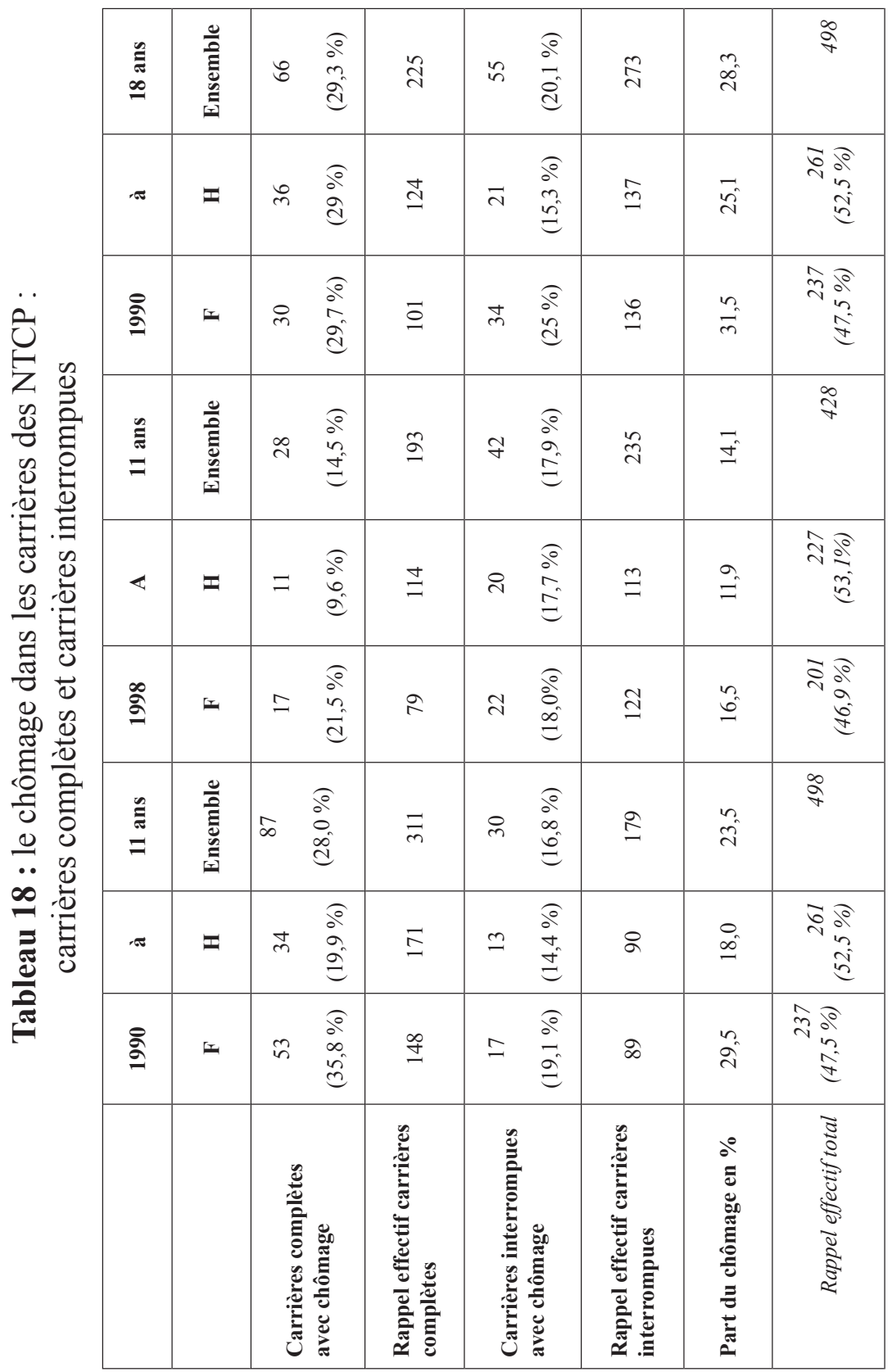


Paradoxalement, ces séquences de chômage déclarées n'apparaissent pas comme des facteurs lourds de discontinuité ou de sortie. La part des séquences de chômage déclarées est proportionnellement plus nombreuse pour les carrières complètes que pour les carrières interrompues. Toutefois apparait une forte inégalité entre les femmes et les hommes : les femmes sont toujours proportionnellement plus nombreuses que les hommes à connaître des périodes de chômage.

Cette présence du chômage s'explique assez largement par la stratégie des entreprises qui, pour diminuer leurs coûts de production dans un environnement publicitaire morose, cherchent à faire diminuer la masse salariale. Elle est également liée aux stratégies individuelles, en particulier des journalistes sous contrats précaires (piges et CDD) qui se déclarent chômeurs pour être indemnisés dans les périodes sans revenus ou à revenus trop faibles (Leteinturier, 2013 ; Albert \& Sonnac, 2014).

Enfin ces périodes d'interruption et les sorties temporaires et/ ou définitives des fichiers de la CCIJP ne sont pas seulement dues à des effets de conjoncture. Il s'agit aussi pour partie de décisions individuelles des journalistes, qui, elles, échappent aux archives de la CCIJP. Métier relationnel par excellence, le journalisme est un excellent poste d'observation des secteurs proches dans le domaine culturel, mais aussi politique ou social, qui offrent également des opportunités d'emploi et de carrière. Cette saisie d'opportunité hors rédaction dans les médias existe puisque l'analyse des séquences préobtention de la carte montre qu'un certain nombre de professionnels des médias non-journalistes basculent sur le journalisme, de façon plus ou moins durable. On peut donc imaginer que l'inverse est possible. C'est ainsi que l'Observatoire des métiers de la presse a observé la part croissante des métiers liés au marketing et à la communication dans les groupes de presse. Certains journalistes peuvent saisir ces opportunités pour rester dans l'entreprise, mais sur un autre poste, qui peut également offrir des avantages en termes de rémunération. Nous avions également montré que l'accès à 
des postes à responsabilités gestionnaires pouvait aussi être une séquence dans les trajectoires des journalistes, certains de ces postes ne permettent pas de conserver la carte de presse.

\section{Une autre approche de la discontinuité : l'ancienneté professionnelle.}

La CCIJP publie chaque année ${ }^{1}$ des données sur le nombre de cartes délivrées avec quelques critères de répartition, qui permettent, cumulées, de mettre en évidence certains phénomènes. C'est ainsi qu'entre 2010 et 2014, la plupart des indicateurs sont à la baisse : le nombre de cartes délivrées $(-6,6$ $\%)$, le nombre de journalistes mensualisés $(-8 \%)$, le nombre d'hommes $(-4,3 \%)$. Continuent de croître les statuts précaires, piges et $\mathrm{CDD},(+3,7 \%)$, et le nombre des journalistes femmes $(+$ $1 \%$ ). Et ces mouvements avaient déjà commencé entre 2005 et 2010.

Tableau 19 : Évolution des principaux indicateurs statistiques de la CCIJP ${ }^{1}$ (CCIJP/ ANR-AMMEJ)

\begin{tabular}{|c|c|c|c|c|c|c|}
\hline & 1981 & 1990 & 2000 & 2005 & 2010 & 2014 \\
\hline $\begin{array}{l}\text { Nombre total } \\
\text { de cartes } \\
\text { délivrées }\end{array}$ & 18041 & 26614 & 32738 & 36503 & 37007 & 34567 \\
\hline $\begin{array}{l}\text { Taux de } \\
\text { croissance } \\
\text { (en \%) }\end{array}$ & & 47,52 & 23,01 & 11,50 & 1,38 & $-6,59$ \\
\hline $\begin{array}{l}\text { Nombre de } \\
1^{\text {ères }} \text { cartes }\end{array}$ & $\mathrm{NC}$ & 2140 & 2280 & 1979 & 1822 & 1748 \\
\hline $\begin{array}{l}\text { Taux de } \\
\text { croissance } \\
\text { (en \%) }\end{array}$ & & & 6,54 & $-13,20$ & $-7,93$ & $-4,06$ \\
\hline $\begin{array}{l}\text { Diplômés } \\
\text { d'Ecoles } \\
\text { reconnues }\end{array}$ & $\mathrm{NC}$ & 72 & 267 & 303 & 225 & 284 \\
\hline $\begin{array}{l}\text { Taux de } \\
\text { croissance } \\
\text { (en \%) }\end{array}$ & & & 73,03 & 11,88 & $-34,67$ & 26,22 \\
\hline
\end{tabular}

1 Voir le site de la CCIJP : http://www.ccijp.net 


\begin{tabular}{|l|c|c|c|c|c|c|}
\hline $\begin{array}{l}\text { Journalistes } \\
\text { mensualisés }\end{array}$ & 15740 & 22861 & 26582 & 29614 & 29558 & 27131 \\
\hline $\begin{array}{l}\text { Taux de } \\
\text { croissance } \\
\text { (en \%) }\end{array}$ & 45,24 & 16,28 & 11,41 & $-0,19$ & $-8,21$ \\
\hline $\begin{array}{l}\text { Pigistes \& } \\
\text { CDD }\end{array}$ & 1968 & 3923 & 6156 & 6889 & 7449 & 7728 \\
\hline $\begin{array}{l}\text { Taux de } \\
\text { croissance } \\
\text { (en\%) }\end{array}$ & 99,34 & 56,92 & 11,91 & 8,13 & 3,75 \\
\hline $\begin{array}{l}\text { Total } \\
\text { Hommes }\end{array}$ & 13628 & 17559 & 19747 & 21028 & 20369 & 19491 \\
\hline $\begin{array}{l}\text { Taux de } \\
\text { croissance } \\
\text { (en\%) }\end{array}$ & 4413 & 9055 & 12991 & 15475 & 16638 & 16826 \\
\hline $\begin{array}{l}\text { Total } \\
\text { Femmes }\end{array}$ & 105,19 & 43,47 & 19,12 & 7,52 & 1,13 \\
\hline $\begin{array}{l}\text { Taux de } \\
\text { croissance } \\
\text { (en\%) }\end{array}$ & 261 & 830 & 1072 & 1582 & 1520 & 1458 \\
\hline Chômeurs & 218,01 & 29,16 & 47,57 & $-3,92$ & $-4,08$ \\
\hline $\begin{array}{l}\text { Taux de } \\
\text { croissance } \\
\text { (en \%) }\end{array}$ & & & & & $-3,13$ \\
\hline
\end{tabular}

Il faut également noter le faible nombre des premières demandes en provenance de journalistes issus des formations reconnues par la profession. Ils sont en moyenne entre 250 et 300 à se présenter devant la CCIJP ce qui correspond environ à 50 à $60 \%$ des effectifs formés par les écoles reconnues. Dès l'entrée sur le marché du travail, certains diplômés en journalisme ne se présentent pas devant la CCIJP, soit qu'ils n'en voient pas l'intérêt soit qu'ils s'orientent vers des secteurs où la carte est inutile et/ou difficile à obtenir.

Ces statistiques annuelles de la CCIJP peuvent donner l'illusion d'une stabilité du groupe des journalistes encartés, avec un flux d'entrée, autour de 2000 personnes par an, qui compense plus ou moins les départs. Toutefois, une analyse plus fine des renouvellements, sorties et premières cartes montre qu'il y a une 
relative stabilité du nombre de cartes délivrées, avec quelques fluctuations récentes; il y a donc une augmentation régulière du nombre des sortants en nombre, mais leur part relative reste stable, autour de $5 \%$ de l'ensemble des encartés.

Tableau 20 : Renouvellements, sorties et premières cartes (en nombre) entre 1990 et 2015 (CCIJP/ANR-AMMEJ)

\begin{tabular}{|l|c|c|c|c|c|}
\hline \multicolumn{1}{|c|}{} & $\mathbf{1 9 9 0}$ & $\mathbf{2 0 0 0}$ & $\mathbf{2 0 0 5}$ & $\mathbf{2 0 1 0}$ & $\mathbf{2 0 1 3}$ \\
\hline $\begin{array}{l}\text { Total cartes } \\
\begin{array}{l}\text { Total renouvel- } \\
\text { lements }\end{array}\end{array}$ & 26614 & 32738 & 36503 & 37007 & 36823 \\
\hline $\begin{array}{l}\text { \% des renouvel- } \\
\text { lements }\end{array}$ & 92 & 30458 & 34524 & 35185 & 35107 \\
\hline $\begin{array}{l}\text { Total première } \\
\text { carte* }\end{array}$ & 2140 & 2280 & 1979 & 1822 & 1716 \\
\hline $\begin{array}{l}\text { \%des nouvelles } \\
\text { cartes }\end{array}$ & 8 & 10 & 1444 & 5 & 4,6 \\
\hline Sortants & & 1444 & 1624 & 2201 & 1905 \\
\hline \% des sortants & 0 & 4,5 & 4,5 & 5,9 & 5,1 \\
\hline
\end{tabular}

La CCIJP a également noué un partenariat avec deux observatoires professionnels, l'Observatoire des métiers de la presse et l'Observatoire des métiers de l'audiovisuel, qui publient chaque année des statistiques générales sur la population encartée. Elles permettent de suivre l'évolution du groupe des encartés, mais ces photographies annuelles ne permettent pas de saisir les carrières des professionnels même si elles fournissent des éléments intéressants d'évolution en termes d'effectif, de féminisation, de statuts, ou concernant la part des différents médias dans l'emploi des journalistes.

\subsection{L'évolution de l'ancienneté professionnelle des journalistes}

L'Institut Français de Presse utilise également ces données globales dans le cadre des enquêtes décennales déjà citées. C'est ainsi que nous avons travaillé sur l'ancienneté professionnelle des journalistes titulaires de la carte de presse entre 1990 et 2012. Son évolution met en évidence une transformation des carrières sur les 20 dernières années. Les anciennetés courtes, moins de 5 
ans se réduisent fortement alors que les anciennetés moyennes, entre 6 et 20 ans évoluent peu. Ce sont les anciennetés les plus longues, supérieures à 20 ans qui augmentent spectaculairement, gagnant 10 points en 22 ans. Ce phénomène est général, car il correspond à celui du baby-boom indiquant la plus grande difficulté d'entrée et surtout de stabilisation des plus jeunes dans le groupe professionnel. On peut donc faire l'hypothèse que ce sont les plus jeunes titulaires de la carte de presse qui quittent le groupe.

Tableau 21 : Répartition des journalistes par tranches d'ancienneté en \% des titulaires de la carte de presse en 1990, 1998 et 2012 (CCIJP / IFP-CARISM)

\begin{tabular}{|l|c|c|c|}
\hline $\begin{array}{c}\text { Ancien- } \\
\text { neté }\end{array}$ & $\begin{array}{c}\text { Journalistes } \\
\text { carte 1990 }\end{array}$ & $\begin{array}{c}\text { Journalistes } \\
\text { carte } 1999\end{array}$ & $\begin{array}{c}\text { Journalistes } \\
\text { carte 2012 }\end{array}$ \\
\hline$=<5$ ans & $\mathbf{3 4 , 9}$ & $\mathbf{2 6 , 3}$ & $\mathbf{2 0 , 3}$ \\
\hline 6/10 ans & $\mathbf{2 1 , 1}$ & $\mathbf{2 1 , 2}$ & $\mathbf{1 9 , 0}$ \\
\hline $11 / 20$ ans & 25,7 & 31,9 & $\mathbf{3 1 , 1}$ \\
\hline$>=21$ ans & $\mathbf{1 8 , 3}$ & $\mathbf{2 0 , 7}$ & $\mathbf{2 9 , 6}$ \\
\hline
\end{tabular}

L'étude de l'ancienneté selon le statut professionnel (salarié ou pigiste) confirme les causes de l'érosion des tranches d'âges les plus jeunes. Si la part des jeunes pigistes reste stable, celle des jeunes salariés baisse fortement (moins de 5 ans). Une très petite minorité de pigistes fait une carrière longue sous ce statut. La majorité des pigistes sont jeunes : leur diminution progressive signifie soit leur intégration comme salariés, soit leur sortie des fichiers de la carte de presse, pour des raisons diverses, mais vraisemblablement pour insuffisance de revenus tirés de l'activité journalistique.

Tableau 22 : répartition par tranches d'ancienneté des pigistes et des salariés en 2012 (CCIJP / IFP - CARISM)

\begin{tabular}{|l|c|c|c|c|}
\cline { 2 - 5 } \multicolumn{1}{c|}{} & $\mathbf{1 9 9 9}$ & $\mathbf{1 9 9 9}$ & $\mathbf{2 0 1 2}$ & $\mathbf{2 0 1 2}$ \\
\hline & Pigistes & Salariés & Pigistes & Salariés \\
\hline$<=\mathbf{5}$ ans & $\mathbf{4 2 , 8}$ & $\mathbf{2 3 , 1}$ & $\mathbf{4 1 , 6}$ & 15,3 \\
\hline $\mathbf{6 - 1 0}$ ans & 20,6 & 21,1 & 19,7 & 19,2 \\
\hline $\mathbf{1 1 - 1 5}$ ans & 16,3 & 19,4 & 14,4 & $\mathbf{1 9 , 9}$ \\
\hline
\end{tabular}




\begin{tabular}{|r|c|c|c|c|}
\hline $\mathbf{1 6 - 2 0}$ ans & 8,8 & 13,9 & 8,1 & 13,9 \\
\hline $\mathbf{2 1 - 2 5}$ ans & 5,5 & 9,9 & 7,9 & 13,7 \\
\hline $\mathbf{2 6 - 3 0}$ ans & 3,1 & 7,4 & 4,6 & 9,3 \\
\hline $\mathbf{3 1 - 3 5}$ ans & 1,4 & 3,8 & 2,0 & 5,9 \\
\hline $\mathbf{3 6 - 4 0}$ ans & 0,8 & 1,2 & 1,3 & 2,3 \\
\hline $\mathbf{> 4 1}$ ans & 0,6 & 0,2 & 0,4 & 0,5 \\
\hline $\begin{array}{r}\text { Total } \\
\text { Effectif }\end{array}$ & 5703 & 24904 & 7672 & 27134 \\
\hline
\end{tabular}

5.2. L'érosion des primo encartés au fil du temps

Le fichier informatique fournit aussi l'année de délivrance de la première carte, il est donc possible de suivre l'évolution des effectifs des « primo-encartés » au fil du temps. .

Tableau 23 : les « sorties » des primo-encartés (CCIJP / IFPCARISM)

\begin{tabular}{|l|c|c|c|c|c|}
\hline $\begin{array}{c}\text { An- } \\
\text { nées }\end{array}$ & $\begin{array}{c}\text { Nombre } \\
\text { de 1 } \\
\text { cartes } \\
\text { délivrées }\end{array}$ & $\begin{array}{c}\text { Effectifs } \\
\text { des } \\
\text { primo- } \\
\text { encartés } \\
\text { encore } \\
\text { présents } \\
\text { en 1999 }\end{array}$ & $\begin{array}{c}\text { \% } \\
\text { d'érosion } \\
\text { en 1998 }\end{array}$ & $\begin{array}{c}\text { Effectifs } \\
\text { des primo- } \\
\text { encartés } \\
\text { encore } \\
\text { présents en } \\
\mathbf{2 0 1 2}\end{array}$ & $\begin{array}{c}\text { \% } \\
\text { d'érosion } \\
\text { en 2012 }\end{array}$ \\
\hline $\mathbf{1 9 8 0}$ & & 686 & & 435 & \\
\hline $\mathbf{1 9 9 0}$ & 2140 & 1455 & 32,1 & 1018 & 52,5 \\
\hline $\mathbf{1 9 9 5}$ & 1624 & 1542 & 5,1 & 1040 & 36,0 \\
\hline $\mathbf{2 0 0 0}$ & 2280 & & & 1668 & 26,8 \\
\hline $\mathbf{2 0 0 5}$ & 1979 & & & 1327 & 32,9 \\
\hline $\mathbf{2 0 1 0}$ & 1822 & & & 1811 & 0,6 \\
\hline
\end{tabular}

Plus de $50 \%$ des nouveaux titulaires de la carte de presse 1990 ne sont plus présents en 2012, puis $36 \%$ des nouveaux entrants de 1995, 26,8\% des nouveaux entrants de 2000, et enfin $32,9 \%$ des nouveaux entrants 2005 . Ces phénomènes d'érosion sont plus ou moins importants selon les années, permettant de repérer les périodes plus délicates pour l'emploi dans la mesure où le critère de l'âge n'est pas le seul facteur explicatif. Ils 
confirment largement ce que montre notre analyse des carrières des nouveaux titulaires de la carte de presse.

\section{Conclusion}

Le constat de la croissance du rythme des sorties des fichiers de la CCIJP conduit à considérer que la faible durée du passage par le journalisme (une moyenne de 15 ans sur l'ensemble des échantillons) situe le journalisme comme une séquence d'activité dans un espace professionnel élargi qu'il est difficile de cerner. Le journalisme étant inscrit dans l'espace des professions culturelles, on peut sans doute faire l'hypothèse que les carrières interrompues s'orientent ponctuellement ou ensuite vers ce champ sans qu'il soit possible de quantifier le phénomène à partir de la seule lecture des dossiers de la CCIJP. Gilles Bastin (2015) s'intéresse à l'activité d'écriture des journalistes, qui les font passer du statut de journaliste à celui d'auteur, conduisant ainsi nombre d'entre eux à ne plus pouvoir obtenir la carte de presse faute de revenus suffisants en journalisme : ils sont devenus auteurs, et ont donc changé de monde professionnel, du point de vue des Commissaires de la CCIJP.

\section{Références}

Bastin, G. (2014). La niche fiscale des journalistes et la morphologie des mondes de l'information, Sur le journalisme, about journalism, Sobre jornalismo, 3, 102-115.

Bastin, G. (2015), Le Paradoxe du paria. Morphologie des mondes de l'information et " persona » des journalistes. Mémoire de synthèse pour l'Habilitation à diriger des recherches. Paris : EHESS.

Devillard, V., \& Rieffel, R. (2001). L'insertion professionnelle des nouveaux journalistes : parcours 1990 - 1998. Dans V. Devillard, et al. (Ed.), Les Journalistes français à l'aube de l'an 2000. Profils et parcours. (pp. 121-158). Paris : Panthéon Assas.

Devillard, V., et al. (Ed.). (2001). Les Journalistes français à l'aube de l'an 2000. Profils et parcours. Paris : Panthéon Assas.

Lallement, M. (2007). Le travail, une sociologie contemporaine. Paris : Gallimard.

Leteinturier, C. (2015). Construire les carrières des journalistes titulaires de la carte de presse. L'exploitation des dossiers de la CCIJP. Dans C. Leteinturier, \& C. Frisque 
(Ed.), Les espaces professionnels des journalistes. Des corpus quantitatifs aux analyses qualitatives, (pp. 173-199). Paris : Panthéon-Assas.

Leteinturier, C. (2014a), Devenir journaliste en presse et le rester : un double défi. Dans C. Leteinturier (Ed.), Les journalistes français et leur environnement : 1990-2012. Le cas de la presse d'information générale et politique. (pp. 13-55). Paris : Panthéon Assas.

Leteinturier, C. (2014b). Des emplois en communication. Quelle place dans les carrières des journalistes français ? Les refus de carte de presse en 2010. Les Cahiers du journalisme, 26, 116-133.

Leteinturier, C. (2012a). La CCIJP et la légitimation des « journalistes professionnels » en France, Sur le journalisme, about journalism, Sobre jornalismo, 2, 34-51.

Leteinturier, C. (2012b). Les carrières de journalistes dans les médias locaux et régionaux : étude de deux cohortes de « nouveaux entrants » (1990-1998). Sciences de la Société, 84/85, 265-290.

Leteinturier, C. (2010). La formation des journalistes français : quelles évolutions ? quels atouts à l'embauche ? Le cas des nouveaux titulaires de la carte de presse 2008. Les Cahiers du journalisme, 21, 110-133.

Marchetti, D., \& Ruellan, D. (2001). Devenir journalistes. Sociologie de l'entrée sur le marché du travail. Rennes : CRAP.

Pierre, A., \& Sonnac N. (2014). La Presse française au défi du numérique, Paris : La Documentation française.

Renoux, J.-L. (2015). Définir les emplois journalistiques dans les médias : statuts, temps de travail, qualifications. Dans C. Leteinturier, \& C. Frisque (Ed.), Les espaces professionnels des journalistes. Des corpus quantitatifs aux analyses qualitatives, (pp. 87-109). Paris : Panthéon-Assas.

Renoux, J. L. (2014). La cartographie des métiers de la presse : prospective et enjeux de controle des emplois dans les rédactions. Dans E. Béthoux, J. V. Koster, S. Monchatre, F. Rey, M. Tallard, \& C. Vincent (Ed.), Emploi, formation, compétences : les régulations de la relation salariale en questions, (pp. 163-175). Toulouse: Octares.

Renoux, J. L. (2011). Les régulations de la relation d'emploi dans les médias français. Dissertation doctorale, Univesrité Paris-Dauphine. Disponible à : (https://tel.archivesouvertes.fr/tel-00669626/document).

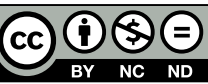

Publié sous la licence Creative Commons

«Attribution - Pas d'Utilisation Commerciale - Pas de Modification 4.0 International» (CC BY-NC-ND) 